\title{
Cochrane Oral Health Group Update
}

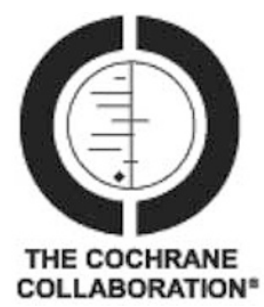

\section{International Symposium 30-31 May 2006}

Evidence for up-to-date clinical dental practice: a review of 10 years of the Cochrane Oral Health Group

Manchester, UK

The Cochrane Oral Health Group is holding a 2-day international Symposium in May 2006 to celebrate their 10 years in Manchester and to promote collaboration in the implementation of research evidence in dental practice.

The symposium will include key plenary presentations, breakout sessions and workshops. Key areas covered will be

- Cochrane: a view from the chair

- How far have we come? Challenges/progress of evidence-based health care in the last 10 years

- The Cochrane Oral Health Group 10 years on, and what is a Cochrane systematic review?

- Evidence-based dentistry: an international perspective

- What is going on in the specialties: caries prevention; restorative/ endodontics; periodontology; implants/prosthetics; orthodontics; oral medicine; dental public health; oral surgery/pain relief

- Where are the gaps and future plans for filling these gaps? What areas need looking at in the different specialties?

- Dissemination of the research evidence

- Implementation of the research evidence

- Barriers to dissemination and implementation in the specialties

- Research methodology

Speakers will include

- James Bader, Research Professor, University of North Carolina, USA

- Iain Chalmers, Editor, James Lind Library, UK

- Jan Clarkson, Director, Effective Dental Practice Programme, Dental Health Services Research Unit, University of Dundee, UK

- Paul Coulthard, Professor of Oral and Maxillofacial Surgery, School of Dentistry, University of Manchester, UK

- Stephen Hancocks, Editor-in-Chief, British Dental Journal, UK

- JA Muir Gray, Director, National Health Service National Electronic Library for Health, UK

- Derek Richards, Director, Centre for Evidence-based Dentistry, UK

- Peter Robinson, Professor, Oral Health and Development, University of Sheffield, UK

- Bill Shaw, Co-ordinating Editor, Cochrane Oral Health Group, UK

- Helen Worthington, Co-ordinating Editor, Cochrane Oral Health Group, UK

- Joanna Zakrzewska, Professor of Pain in Relation to Oral Medicine, Queen Mary's School of Medicine and Dentistry, UK

Further details can be found at www.cochrane-oral.man.ac.uk/ symposium.html

\section{Cochrane Library 2006 issue 1}

January saw the release of the latest update of the Cochrane library, in the Cochrane Database of Systematic Reviews 2006, Issue 1. There were updates of three existing Cochrane Oral Health Group reviews:

1. Littlewood SJ, Millett DT, Doubleday B, Bearn DR, Worthington HV. Retention procedures for stabilising tooth position after treatment with orthodontic braces.

2. Esposito M, Grusovin MG, Worthington HV, Coulthard P Interventions for replacing missing teeth: bone augmentation techniques for dental implant treatment.

3. Matharu L, Ashley PF. Sedation of anxious children undergoing dental treatment.

These are briefly summarised below, together will other relevant Cochrane reviews not covered in detail in the journal. In addition to these updated reviews, two new protocols have been added (again, to the Cochrane Database of Systematic Reviews 2006 Issue 1):

1. Craane B, De Laat A, Dijkstra PU, Stappaerts K, Stegenga B. Physical therapy for the management of patients with temporomandibular disorders and related pain.

2. Lu H, Koh H, Rasines Alcaraz MG, Schmidlin P, Davis D. Direct composite resin fillings versus amalgam fillings for permanent or adult posterior teeth.

Retention procedures for stabilising tooth position after treatment with orthodontic braces (review update)

Littlewood SJ, Millett DT, Doubleday B, Bearn DR, Worthington HV.

Retention procedures for stabilising tooth position after treatment with orthodontic braces. In the Cochrane Database of Systematic Reviews 2006, issue 1.

There is insufficient evidence about the effects of different types of retainers to keep teeth in position after the use of orthodontic braces.

Retention is the phase of orthodontic treatment that attempts to keep teeth in the corrected positions after orthodontic braces. Without a phase of retention there is a tendency for the teeth to return to their initial position (relapse). To prevent relapse, almost every patient who has orthodontic treatment will require some type of retention. There is a lack of robust evidence on which to base clinical practice in this area. This review found weak, unreliable evidence that a simple surgical procedure, combined with a retainer, is better than a retainer alone at keeping teeth in the corrected positions after orthodontic braces are removed. There is an urgent need for high-quality randomised controlled trials in this crucial area of orthodontic practice. 
Bone augmentation techniques for dental implant treatment (review update)

Esposito M, Grusovin MG, Worthington HV, Coulthard P. Interventions for replacing missing teeth: bone augmentation techniques for dental implant treatment. In the Cochrane Database of Systematic Reviews 2006, issue 1.

Some patients have insufficient bone to place dental implants but there are many surgical techniques to increase the bone volume and thus make implant treatment possible.

Short implants are more effective and cause fewer complications than conventional implants placed in thin mandibles augmented with bone from the hip. Bone substitutes (Bio-Oss, Geistlich Pharmaceutical, Wolhusen, Switzerland or Cerasorb Curasan AG, Kleinostheim, Germany) might be used instead of autogenous bone graft to fill large maxillary sinuses. Bone can be regenerated in a vertical direction using both the osteodistraction and the guided bone regeneration techniques, but it is unclear if any one technique is preferable. There is not enough evidence supporting or refuting the need for augmentation procedures when single extracted teeth are immediately replaced with dental implants, nor is it known whether any augmentation procedure is better than the others. There is not enough evidence to demonstrate superiority of any particular technique for regenerating bone around exposed implants.

\section{Sedation of anxious children undergoing dental treatment} (review update)

\section{Matharu L, Ashley PF.}

Sedation of anxious children undergoing dental treatment. In the Cochrane Database of Systematic Reviews 2006, issue 1.

Fear of the dentist or behaviour management problems can result in a child's tooth decay going untreated. Behavioural techniques play an important role in managing anxiety but some children still find it difficult to tolerate dental treatment and may require sedation. This review examined the effectiveness of drugs that sedate a child whilst keeping them conscious. Owing to the poor quality of the research, the review was unable to determine which drugs or methods of sedation are the best for managing a child's anxiety or behaviour.

\section{Oral appliances for obstructive sleep apnoea (review update)}

Lim J, Lasserson TJ, Fleetham J, Wright J.

Oral appliances for obstructive sleep apnoea. In the Cochrane Database of Systematic Reviews 2006, issue 1.

This is a review of the oral appliances used for treating sleepiness, reporting quality of life and markers of sleep disruption in people with obstructive sleep apnoea/hypopnoea (OSAH).
OSAH is characterised by recurrent episodes of partial or complete upper-airway obstruction during sleep, leading to a variety of symptoms including excessive daytime sleepiness. The current firstchoice therapy is continuous positive airways pressure (CPAP) which keeps the upper airway unobstructed during sleep but this treatment can be difficult for some patients to tolerate and comply with on a long-term basis.

Oral appliances (OA) are now widely used as an alternative to CPAP therapy. They are designed to keep the upper airway open by either advancing the lower jaw forward or by keeping the mouth open during sleep. This review found that OA should not be considered as first-choice therapy for OSAH where symptoms and sleep disruption are severe. There has not been enough research examining the effects of OA compared with CPAP in terms of symptoms and quality of life. Although CPAP was clearly more effective at reducing the disruption to sleep, some people with OSAH may prefer using OA if they are found to be tolerable and more convenient than CPAP. When an active OA was compared with an inactive $\mathrm{OA}$, there were improvements in daytime sleepiness and OSAH severity. OA may be more effective than corrective upper airway surgery. Further research should consider whether people with more severe symptoms respond in a similar way to those patients represented in the studies included in the review.

\section{Getting Involved}

The Cochrane Oral Health Group has over 624 members from 40 different countries around the world and welcomes new members. If you would like to get involved, you can find out more at the website (www.cochrane-oral.man.ac.uk/getinvolved.html) or contact the group at its editorial base:

Cochrane Oral Health Group, MANDEC, School of Dentistry, University of Manchester, Higher Cambridge Street, Manchester M15 6FH, UK (Tel: + 44 (0) 161275 7818; Fax: + 44 (0) 161275 7815).

Review Group Co-ordinator (E-mail: emma.tavender@manchester. ac.uk)

Assistant Review Group Co-ordinator (E-mail: luisa.fernandez@ manchester.ac.uk)

Trials Search Co-ordinator (E-mail: sylvia.r.bickley@manchester. ac.uk)

Evidence-Based Dentistry (2006) 7, 20-21.

doi:10.1038/sj.ebd.6400380 